\title{
Anonymat et compensation financière du don de gamètes et d'embryons en Belgique
}

\author{
Bernard LEJEUNE
}

Centre de Procréation assistée Centre Hospitalier Inter Régional Edith Cavell, Bruxelles

\section{RESUME}

Une législation, relative à la pratique de la procréation médicalement assistée (PMA) et à la destination des gamètes et des embryons surnuméraires, a été adoptée en 2007 et est entrée en vigueur fin juillet 2007 en Belgique.

Elle ne change que peu de choses à la pratique courante sauf qu'elle limite drastiquement le nombre de bébés que l'on peut engendrer par donneur de gamètes.

En Belgique, le don de sperme est en principe anonyme mais ne l'est pas obligatoirement : dans le cas où donneur et receveuse se présentent ensemble, font la demande d'un don direct et signent l'autorisation de procéder de la sorte, la procédure est recevable.

Le don de sperme est gratuit mais il est autorisé d'accorder un dédommagement raisonnable au donneur pour les frais engagés et la perte de revenu éventuelle.

Le don d'ovocytes est en principe anonyme et gratuit également mais il n'est pas interdit de procéder à un don dirigé (non anonyme) si l'ensemble des parties en signent l'autorisation. Dans ce cas, donneuse et receveuse se présentent ensemble.

Il est également autorisé d'accorder un dédommagement à la donneuse mais il n'y a pas d'accord jusqu'à présent sur le montant acceptable. En tenant compte des nécessités du traitement, il serait juste de prévoir une incapacité de travail de 10 à 15 jours, donc une perte de revenu équivalente que l'on devrait compenser par un dédommagement de 500 à 1000 euros, des déplacements, voire un séjour au voisinage du Centre de PMA pendant cette période pouvant également se chiffrer à 500 euros à 1000 euros. Le dédommagement justifié se monterait donc à 1000, voire 2000 euros. Ce montant semblait excessif jusqu'à présent mais les sensibilités pourraient changer. II n'en reste pas moins vrai que les donneuses volontaires spontanées pour un don gracieux et anonyme sont rares.

Certains centres procèdent à une permutation des donneuses pour assurer l'anonymat.

Le don d'embryons est anonyme. Les embryons ne peuvent être commercialisés mais le Centre de PMA doit assurer la sécurité sanitaire de ces embryons et leur gestion au bout des 5 années légales de conservation. Ce qui a aussi un coût.

Mots clés : don de gamètes, don d'ovocytes, don de sperme, don d'embryons, anonymat, dédommagement

\section{INTRODUCTION}

Une législation relative à la procréation médicalement assistée et à la destination des embryons surnuméraires et des gamètes a été adoptée en 2007 et est entrée en vigueur fin juillet 2007 en Belgique.

Elle ne change que peu de choses à la pratique courante sauf qu'elle limite drastiquement le nombre de bébés que l'on peut engendrer par donneur de gamètes.

\section{PRINCIPES GENERAUX}

\section{Limites d'âge}

Le prélèvement de gamètes est autorisé entre 18 et 45 ans.

Exceptionnellement, le prélèvement de gamètes sur un mineur peut être autorisé sur indication médicale.

Les FIV, les donneuses d'ovocytes, et les donneurs de sperme sont donc concernés par cette limite.

La demande d'insémination ou d'implantation d'embryons est autorisée entre 18 et $\mathbf{4 5}$ ans. La mise en

\section{Correspondance :}

Dr Bernard LEJEUNE - Centre de Procréation assistée, Centre Hospitalier Inter Régional Edith Cavell, 32 rue E Cavell, $B$ -

1180 Bruxelles - Tel 3223404083 - Fax 3223471996 -

Email pma@chirec.be 
route d'un traitement, tant pour une FIV que pour un don d'ovocytes, ou un don d'embryons ne peut donc plus se faire après 45 ans.

L'implantation d'embryons est autorisée jusqu'à $\mathbf{4 7}$ ans. Cela concerne donc uniquement les couples ayant encore des embryons congelés, provenant d'un essai de FIV ou de don d'ovocytes réalisé avant l'âge de 45 ans.

En cas de refus de prise en traitement par le Centre, celuici doit être signifié par écrit dans le mois, au couple ou à la femme demandeuse, précisant la raison médicale ou la clause de conscience motivant le refus.

\section{DON D'EMBRYONS}

Le don d'embryons est anonyme et gratuit de la part du couple donneur. L'anonymat est donc également garanti tant au couple donneur qu'au couple receveur.

La commercialisation des embryons est interdite (mais le coût de la gestion et du contrôle sanitaire doit être pris en compte).

Le Centre doit vérifier la sécurité sanitaire des embryons donnés.

II ne peut y avoir de sélection de sexe ni d'autres caractères mais l'appariement phénotypique est autorisé.

II est interdit de transférer simultanément des embryons provenant de donneurs différents.

Enfin, les embryons d'un même couple donneur ne peuvent conduire à la naissance d'enfants chez plus de 6 femmes différentes.

\section{RECEVEUR D'EMBRYONS}

Une demande du bénéfice d'un don d'embryons doit être adressée au Centre par lettre recommandée, signée par les deux membres du couple, ou par la femme seule s'il n'existe pas de couple.

La réponse du Centre doit être dans les deux mois, proposant la convention ad hoc ou précisant les motif de refus (raison médicale ou de conscience, et donnant le cas échéant les coordonnées d'autres Centres).

Une convention doit être établie entre le Centre et le couple ou la femme seule, receveur d'embryons, précisant : l'identification du Centre, l'identité, date de naissance et adresse du receveur (couple ou femme seule), l'acceptation du recours au don d'embryons anonyme.

La convention doit être établie en deux exemplaires, signés par le(s) auteur(s) du projet parental et contre signés par un représentant autorisé du Centre ; un exemplaire est remis au couple, l'autre est conservé par le Centre.

Un avenant à la Convention peut être établi à tout moment.

\section{DONNEUR DE GAMETES}

Les gamètes peuvent être prélevés/déposés en vue d'un projet parental, d'un don de gamètes, d'un protocole de recherche.
Le délai de conservation est de 10 ans dans le cadre d'un projet parental.

Il peut être réduit à la demande de la personne qui a sollicité la conservation.

En raison de circonstances exceptionnelles, le délai de conservation peut être prolongé à la demande du dépositaire et avec l'accord du Centre ; sinon les dispositions légales seront appliquées (destruction, protocole de recherche ou don selon le choix convenu au départ avec le dépositaire).

Le Centre doit fournir soutien psychologique et informations sur le mode de prélèvement, les risques, le délai de conservation et le devenir des gamètes.

Une convention doit être établie entre le Centre et le donneur de gamètes précisant :

\section{L'identification du Centre.}

L'identité, date de naissance et adresse du donneur.

L'engagement du donneur à réaliser les examens nécessaires pour établir la sécurité sanitaire des gamètes fournis.

La destination initiale des gamètes (projet parental au sein du couple, don spécifié ou don anonyme, protocole de recherche).

Dans le cadre d'un projet parental, la destination des gamètes en cas de dissolution du couple (décès, divorce, mésentente insoluble sur la destination des gamètes) et à l'échéance du délai de conservation doit y être précisée.

La convention doit être établie en deux exemplaires, signés par les auteurs du projet parental et contresignés par un représentant autorisé du Centre ; un exemplaire est remis au couple, l'autre est conservé par le Centre.

Un avenant à la Convention peut être établi à tout moment mais une fois la procédure de don de gamètes engagée, le don est irrévocable.

Avant toute insémination de gamètes au sein d'un couple, le couple doit réitérer par écrit son consentement à l'insémination.

L'insémination post mortem est autorisée pour autant qu'elle ait été expressément prévue dans la convention et qu'elle ait lieu dans un délai de 6 mois minimum et 2 ans maximum après le décès.

\section{Dispositions :}

Le don de gamètes doit être gratuit mais des indemnités de déplacement, de pertes de salaire, de frais d'hospitalisation peuvent être accordées au donneur (donneuse).

Le Centre doit s'assurer de la sécurité sanitaire des gamètes et les donneurs doivent s'engager à réaliser les examens nécessaires à cet égard.

II ne peut exister de commercialisation des gamètes (mais le coût de la gestion et du contrôle sanitaire des paillettes doit être pris en compte).

Le don est en principe anonyme mais le don non anonyme 
est autorisé s'il s'appuie sur un accord écrit entre donneur et receveur.

II ne peut y avoir de sélection de sexe ou d'intention eugénique dans le recours au don si ce n'est un motif médical (maladie liée au sexe).

L'appariement des phénotypes des donneurs et receveurs est autorisé.

II est interdit d'inséminer simultanément avec des gamètes provenant de donneurs différents.

Les gamètes d'un même donneur ne peuvent conduire à la naissance d'enfants chez plus de 6 femmes différentes.

La conservation des données médicales et phénotypiques du donneur et de la convention entre le Centre et le donneur, est assurée par le Centre. Seules les données médicales peuvent être transmises au médecin de la personne conçue par le don, si son état de santé le requière.

\section{RECEVEUR DE GAMETES}

Une demande du bénéfice d'un don de gamètes par lettre recommandée adressée au Centre, signée par les deux membres du couple, ou par la femme seule s'il n'existe pas de couple.

La réponse du Centre dans les deux mois proposant la convention ad hoc ou précisant les motif de refus (raison médicale ou de conscience, et donnant le cas échéant les coordonnées d'autres Centres).

Une convention doit être établie entre le Centre et le receveur de gamètes précisant :

- L'identification du Centre.

- L'identité, date de naissance et adresse du receveur.

- L'acceptation du recours au don de gamètes (en précisant s'il s'agit d'un don anonyme ou d'un don spécifié).

La convention doit être établie en deux exemplaires, signés par le(s) auteur(s) du projet parental et contre signés par un représentant autorisé du Centre ; un exemplaire est remis au couple, l'autre est conservé par le Centre.

Un avenant à la Convention peut être établi à tout moment.

\section{CONCLUSION}

En Belgique, le don de sperme est en principe anonyme mais ne l'est pas obligatoirement : dans le cas où donneur et receveuse se présentent ensemble, font la demande d'un don direct et signent l'autorisation de procéder de la sorte, la procédure est recevable.

Le don de sperme est gratuit mais il est autorisé d'accorder un dédommagement raisonnable au donneur pour les frais engagés et la perte de revenu éventuelle.

Le don d'ovocytes est en principe anonyme et gratuit également mais il n'est pas interdit de procéder à un don dirigé (non anonyme) si l'ensemble des parties en signent l'autorisation. Dans ce cas, donneuse et receveuse se présentent ensemble.
Il est également autorisé d'accorder un dédommagement à la donneuse mais il n'y a pas d'accord jusqu'à présent sur le montant acceptable. En tenant compte des nécessités du traitement, il serait juste de prévoir une incapacité de travail de 10 à 15 jours, donc une perte de revenu équivalente que l'on devrait compenser par un dédommagement de 500 à 1000 euros, des déplacements voire un séjour au voisinage du Centre de PMA pendant cette période pouvant également se chiffrer à 500 à 1000 euros. Le dédommagement justifié se monterait donc à 1000 voire 2000 euros. Ce montant semblait excessif jusqu'à présent mais les sensibilités pourraient changer. II n'en reste pas moins vrai que les donneuses volontaires spontanées pour un don gracieux et anonyme sont rares.

Certains centres procèdent à une permutation des donneuses pour assurer l'anonymat.

Le don d'embryons est anonyme. Les embryons ne peuvent être commercialisés mais le Centre de PMA doit assurer la sécurité sanitaire de ces embryons et leur gestion au bout des 5 années légales de conservation. Ce qui a aussi un coût.

\section{REFERENCES}

Moniteur belge 17 juillet 2007 : loi relative à la procréation médicalement assistée et à la destination des embryons surnuméraires et des gamètes. www.moniteur.be

Manuscrit reçu : février 2008 ; accepté mars 2008.

Communication présentée lors du XXIVème Congrès de la SALF, Colmar décembre 2007.

\section{ABSTRACT}

Anonymity and financial compensation for gamete and embryo donation in Belgium

\section{Bernard LEJEUNE}

Belgian law concerning medically assisted procreation (MAP) and use of gametes and supernumerary embryos was adopted in 2007 and entered into force at the end of July 2007. It has only a limited impact on routine clinical practice, but dramatically limits the number of pregnancies that can be achieved by gamete donation.

In Belgium, sperm donation is theoretically anonymous, but anonymity is not mandatory: when the donor and recipient attend the clinic together, request direct donation and sign an agreement, this procedure is acceptable. 
Sperm donation is free but it is permitted to provide reasonable compensation to the donor for any expenses and loss of income.

Ooocyte donation is also free and theoretically anonymous, but directed (non-anonymous) donation is not prohibited when all parties sign an agreement. In this case, the donor and recipient attend the clinic together.

It is also permitted to provide compensation to the donor, but no agreement has been reached concerning the acceptable amount. By taking into account the need for treatment, it would be reasonable to provide for 10 to 15 days off work and therefore an equivalent loss of income that could be compensated by 500 to 1000 euros, travel expenses, or even accommodation close to the MAP clinic during this period, which could also represent 500 to 1000 euros. Justified compensation would therefore represent 1000 or even 2000 euros. This amount appeared to be excessive up until now, but opinions tend to change. It is nevertheless true that spontaneous volunteer donors for free and anonymous oocyte donation are rare.

Some centres perform donor randomisation to ensure anonymity.

Embryo donation is anonymous. Embryos cannot be sold, but the MAP clinic must ensure the health security of these embryos and their management after the 5-year legal storage period, which also has a certain cost.

Key words: gamete donation, oocyte donation, sperm donation, embryo donation, anonymity, compensation 